\title{
MicroRNA-212 Inhibits Osteosarcoma Cells Proliferation and Invasion by Down- Regulation of Sox4
}

\author{
Xiao-Ji Luo Da-Gang Tang Tian-Le Gao Yan-Liang Zhang Miao Wang \\ Zheng-Xue Quan Jin Chen \\ Department of Orthopedics, the First Affiliated Hospital of Chongqing Medical University, Chongqing, \\ P. R. China
}

\section{Key Words}

Osteosarcoma $\cdot$ MicroRNA $\cdot \operatorname{miR}-212 \cdot \mathrm{SOX} 4$

\begin{abstract}
Background: Multiple MicroRNAs (miRNAs) have been identified in the development and progression of osteosarcoma. However, the expression and roles of miR-212 in osteosarcoma remain largely undefined. Methods: Real-time PCR assays were used to detect the expression of miR-212 in human osteosarcoma tissues. MiR-212 mimics were introduced into MG63 and U2OS cells. Bioinformatic prediction was used to identify the potential targets of miR212. Protein expression analysis, luciferase assays and rescue assays were used to confirm the substrate of miR-212. Results: miR-212 was significantly down-regulated in human osteosarcoma tissues, compared with adjacent normal tissues. Introduction of miR-212 mimics into MG63 and U2OS cells inhibited cell proliferation and invasion. Besides, miR-212 overexpression could also inhibit tumor growth in the nude mice. Additionally, bioinformatic prediction suggested that the sex-determining region Y-box 4 (Sox4) is a target gene of miR212. Sox4 inhibition phenocopied the roles of miR-212, while restored expression of Sox4 dampened miR-212-mediated suppression of tumor progression. Conclusion: The miR-212/ Sox4 interaction plays an important role of in the osteosarcoma progression.
\end{abstract}

Copyright (C) 2014 S. Karger AG, Basel

\section{Introduction}

Osteosarcoma is the most frequent malignant bone tumor in children and young adults, with a peak incidence at the age of 15-19 years [1, 2]. Therefore, to identify novel mechanisms may help to develop effective strategies for the diagnosis, treatment and prognosis of osteosarcoma. 
MicroRNAs (miRNAs), a class of small non-coding RNA molecules, play pivotal roles in variety of biological events, including development, cell proliferation and differentiation [3-5]. Previous studies have shown that multiple miRNAs are differentially expressed in human cancers, including osteosarcoma [6-8], providing new insights into the complex genetic mechanisms of cancer progression. For instance, miR-16 was shown to inhibit cell proliferation by targeting IGF1R and the Raf1-MEK1/2-ERK1/2 pathway in osteosarcoma [9], while miR-802 promoted osteosarcoma cell proliferation by down-regulation of cellcycle inhibitor, p27 [10].

It has been shown that miR-212 inhibited proliferation of gastric cancer by directly repressing retinoblastoma binding protein 2 [11]. Besides, miR-212 inhibited cell proliferation and induced cellular senescence in hepatocellular carcinoma through suppression of histone demethylase retinoblastoma binding protein 2 [12]. Moreover, miR212 displayed tumor-promoting properties in non-small cell lung cancer cells by targeting the hedgehog pathway receptor PTCH1 [13]. However, until now, the biological functions of miR-212 in osteosarcoma remain unknown.

In the present study, we found that miR-212 was significantly down-regulated in osteosarcoma tissues, and further revealed that overexpression of miR-212 potently inhibited osteosarcoma progression in vitro and in vivo.

\section{Materials and Methods}

\section{Clinical tissue samples}

28 pairs of osteosarcoma tissues and adjacent normal tissues were collected from routine therapeutic surgery at our department. All samples were obtained with informed consent and approved by the hospital institutional review board.

\section{Cell culture}

Osteosarcoma cell lines (MG63 and U20S cells) were purchased from American Type Culture Collection (Rockville, MD, USA). Cells were culture in RPMI 1640 medium (GIBCO, Shanghai, China) supplemented with $10 \%$ fetal bovine serum (GIBCO). Cultures were incubated at $37^{\circ} \mathrm{C}$ with $5 \% \mathrm{CO} 2$ in a humidified incubator.

\section{RNA isolation and quantitative real-time PCR}

Total RNA from tissue samples and cell lines was harvested using the RNA Isolation Kit (Ambion, USA). Expression of mature miRNAs was assayed using Taqman MicroRNA Assay (Applied Biosystems) specific for hsa-miR-212. Quantitative real-time PCR was performed by using an Applied Biosystems 7900 Realtime PCR System and a TaqMan Universal PCR Master Mix. All the primers were obtained from the TaqMan miRNA Assays. Small nuclear U6 snRNA (Applied Biosystems) was used as an internal control. Differences in gene expression, expressed as fold-changes, were calculated using the $2^{-\Delta \Delta C t}$ method.

miR-212 mimics, antisense and transfection

Human miR-212 mimics, antisense or negative controls were obtained from Genepharm Company (Shanghai). For transfection, cells were cultured in a 6 well plate and transiently transfected at 70-80\% confluence using the LipofectamineTM 2000 reagent (Invitrogen, CA, USA) as per the manufacturer's instructions.

BrdU and cell invasion assays

A cell proliferation enzyme-linked immunosorbent assay (BrdU kit; Beyotime) was used to analyze the incorporation of BrdU during DNA synthesis following the manufacturer's protocols. All experiments were performed in triplicate. Absorbance was measured at $450 \mathrm{~nm}$ in the Spectra Max 190 ELISA reader (Molecular Devices, Sunnyvale, CA). For cell invasion assays, cells were analyzed using extracellular matrixcoated invasion chambers (Millipore, CA, USA), and quantitated with a colorimetric microplate reader at $570 \mathrm{~nm}$, according to the manufacturer's instructions. 
Luo et al.: mir212 Targets SOX4

Cell-cycle analysis

Cells were labeled for 20 min with propidium iodide and immediately analyzed by flow cytometry. Histograms represent the percentage of cells in each phase of the cell cycle (G0/G1, S and G2/M).

Tumor growth assay

Male BALB/c nude mice aged 4 weeks were purchased from Shanghai Laboratory Animal Company (SLAC, Shanghai). $2.5 \times 10^{5}$ MG62 cells stably expressing miR-212 or negative control were injected subcutaneously to the skin under the front legs of the mouse. The mice were observed over 4weeks for tumor formation. After the mice were sacrificed, the tumors were recovered and the wet weights of each tumor were determined.

\section{Western blot}

Cells or tissues were harvested and lysed with lysis buffer $(150 \mathrm{mM} \mathrm{NaCl}, 50 \mathrm{mM}$ Tris- $\mathrm{HCl}, 1 \% \mathrm{NP}-$ 40, pH7.5). Proteins were quantified and separated by $8 \%$ SDS-Agarose gel, transferred to NC membrane (Amersham Bioscience, Buckinghamshire, U.K.). After blocking with 10\% nonfat milk in PBS, membranes were immunoblotted with antibodies as indicated, followed by HRP-linked secondary antibodies (Cell Signaling). The signals were detected by SuperSignal West Pico Chemiluminescent Substrate kit (Pierce, Rockford, IL) according to manufacturer's instructions. Anti-SOX4 antibody was purchased from Abcam Company (USA). Protein levels were normalized to total GAPDH, using a mouse anti-GAPDH antibody (Santa Cruz, USA).

Luciferase reporter assay

3'-UTR of human SOX4 gene that were predicted to interact with miR-212 were synthesized and inserted into pMir-Report (Ambion, USA), yielding pMir-Report-SOX4. Mutations within potential miR-212 binding sites were generated by nucleotide replacement of wild type sequence to inhibit miR-212 binding. Cells were transfected with the pMir-Report vectors containing the 3'-UTR variants, and miR-212 mimics for 36 hours. The pRL-SV40 vector (Promega, USA) carrying the Renilla luciferase gene was used as an internal control to normalize the transfection efficiency. Luciferase values were determined using the DualLuciferase Reporter Assay System (Promega).

\section{Statistics}

Data are expressed as the mean \pm SEM from at least four separate experiments. Differences between groups were analyzed using Student's t-test or ONE Way ANOVA analysis. A value of $\mathrm{p}<0.05$ was considered statistically significant.

\section{Results}

\section{Down-regulation of miR-212 in osteosarcoma tissues}

Firstly, we analyzed the expression of miR-212 in 28 pairs of human osteosarcoma tissues and pair-matched adjacent noncancerous tissues. As shown in Figure 1, results from real-time PCR analysis showed that miR-212 was significantly down-regulated in osteosarcoma tissues in comparison with the adjacent normal tissues (Fig. 1).

Fig. 1. Expression levels of miR-212 in osteosarcoma tissues. miR-212 expression was determined by real-time PCR in human osteosarcoma tissues and adjacent noncancerous tissues (Normal). ${ }^{* * *} \mathrm{P}<0.001$ between two groups.

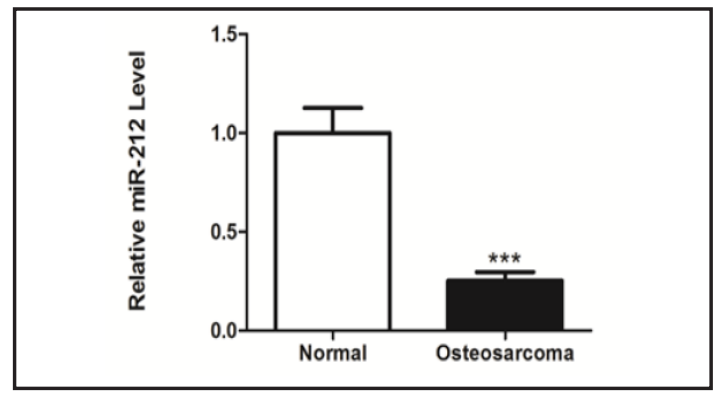


A

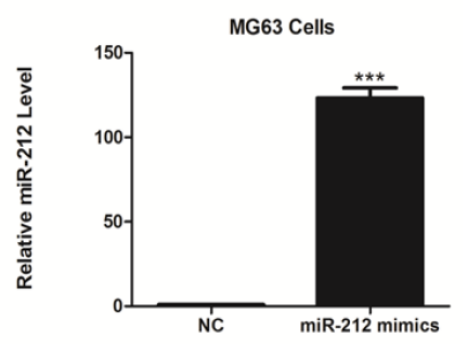

C

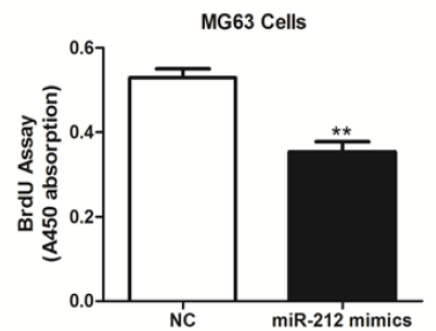

E

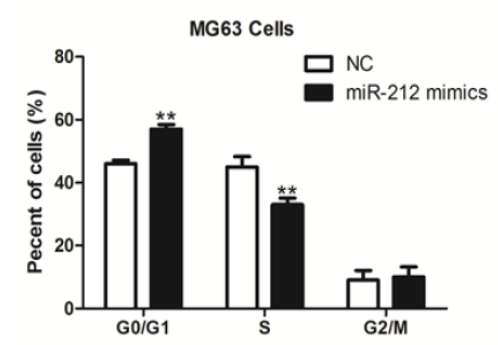

G

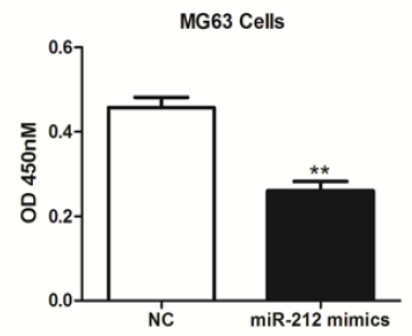

B

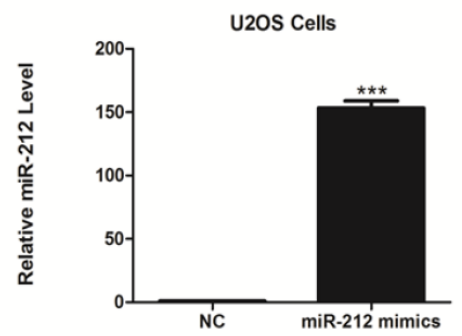

D

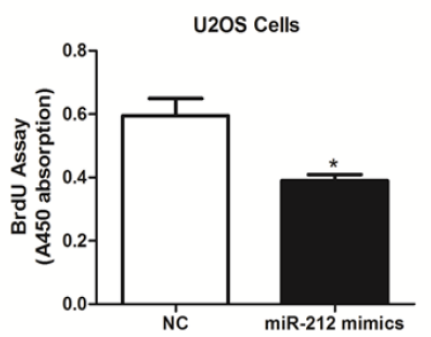

$\mathbf{F}$

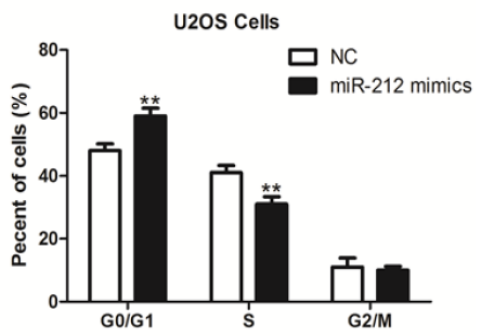

H

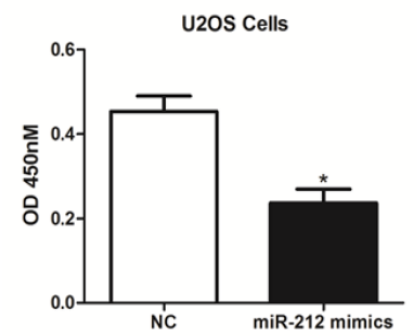

Fig. 2. miR-212 mimics inhibits osteosarcoma cell proliferation. (A-B) Expression levels of miR-212 were determined by real-time PCR in MG63 (A) and U20S (B) cells transfected with miR-212 mimics or negative controls (NC) for $24 \mathrm{hr}$. (C-D) The cell proliferative potential (BrdU) was determined in MG63 (C) and U20S (D) cells transfected with miR-212 mimics or negative control (NC). (E-F) The cell-cycle phase of MG63 (E) and U2OS (F) cells transfected with miR-212 mimics or negative controls (NC) were analyzed by flow cytometry. (G-H) The cell invasion assays were performed in MG63 (G) and U2OS (H) cells transfected with miR-212 mimics or negative control (NC). After transfected with miR-212 mimics or NC for $24 \mathrm{hr}$, cells were seeded in ECM gel-coated Transwell culture chambers and incubated for another $24 \mathrm{hr}$, and then the invasion assays were measured. ${ }^{*} \mathrm{P}<0.05,{ }^{* *} \mathrm{P}<0.01,{ }^{* * *} \mathrm{P}<0.001$ between two groups.

miR-212 inhibits cell proliferation and invasion in vitro

Next, to clarify the roles of miR-212 in osteosarcoma, its mimics or negative controls (NC) were transfected into MG63 and U2OS cells (Fig. 2A-2B). As a result, miR-212 mimics 
A

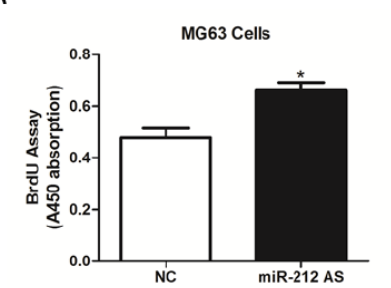

D

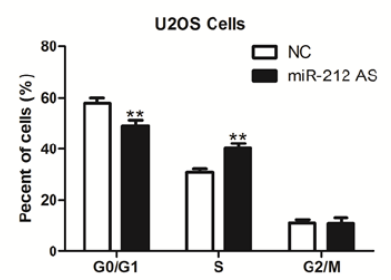

B

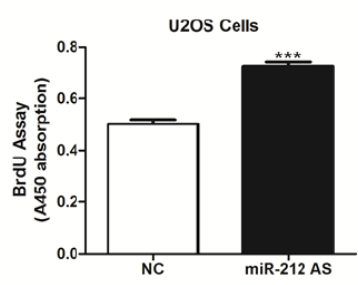

E

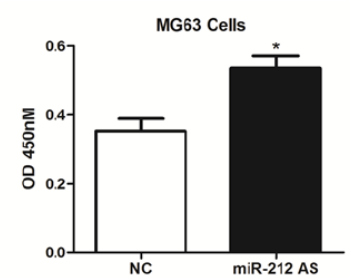

C

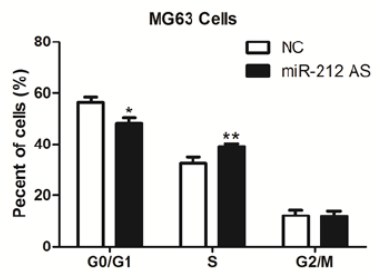

$\mathbf{F}$

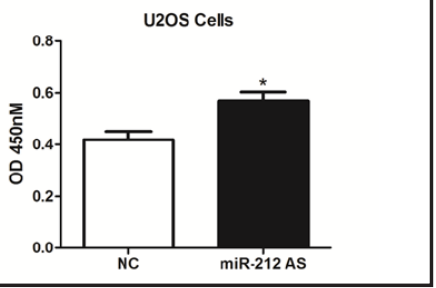

Fig. 3. miR-212 antisense promotes osteosarcoma cell proliferation and invasion. (A-B) The proliferative potential (BrdU) assays were determined in MG63 (A) and U2OS (B) cells transfected with miR-212 antisense (AS) or negative controls (NC) for $24 \mathrm{hr}$. (C-D) The cell-cycle phase of MG63 (C) and U20S (D) cells transfected with miR-212 antisense (AS) or negative controls (NC) were analyzed by flow cytometry. (E-F) The cell invasion assays were performed in MG63 (E) and U2OS (F) cells transfected with miR-212 antisense (AS) or negative control (NC). ${ }^{*} \mathrm{P}<0.05,{ }^{* *} \mathrm{P}<0.01,{ }^{* * *} \mathrm{P}<0.001$ between two groups.

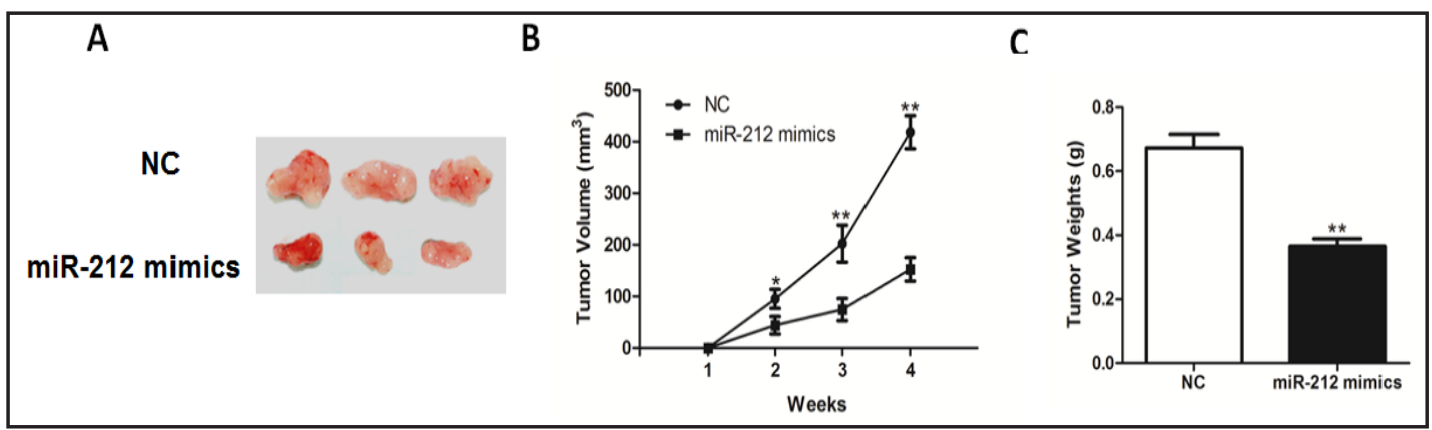

Fig. 4. miR-212 overexpression inhibits tumor growth in vivo. (A-C) MG63 cells stably transfected with miR212 mimics or negative controls $(\mathrm{NC})$ were injected into nude mice ( $\mathrm{n}=5$ for each group) and followed up for tumorigenesis. Representative images (A), growth curve of tumor volumes (B) and tumor weights (C) were taken 4 weeks after injection. ${ }^{*} \mathrm{P}<0.05,{ }^{* *} \mathrm{P}<0.01$ between two groups.

significantly inhibited cell proliferation as shown by BrdU incorporation assays (Fig. 2C2D). In agreement, cell-cycle analysis revealed that miR-212 overexpressing cells had a significantly increased percentage of cells in the G1/G0 phase and lower percentage of cells in the S phase, compared to negative control-transfected cells (Fig. 2E-2F). Moreover, we discovered that ectopic expression of miR-212 mimics inhibited the invasion of both cells (Fig. 2G-2H). In addition, MG63 and U2OS cells were further transfected with miR-212 antisense. As expected, we found that miR-212 antisense promoted the growth and invasion of osteosarcoma cells, compared to negative controls (Fig. 3A-3F). Taken together, our results indicate that miR-212 could suppress cell proliferation and invasion in osteosarcoma in vitro.

miR-212 overexpression inhibits tumor growth in vivo

To further determine the roles of miR-212, MG63 cells with stable overexpression of miR-212 mimics were generated and injected subcutaneously to the skin under the front legs of the nude mice. The tumor growth was closely monitored for another 4 weeks. As a 


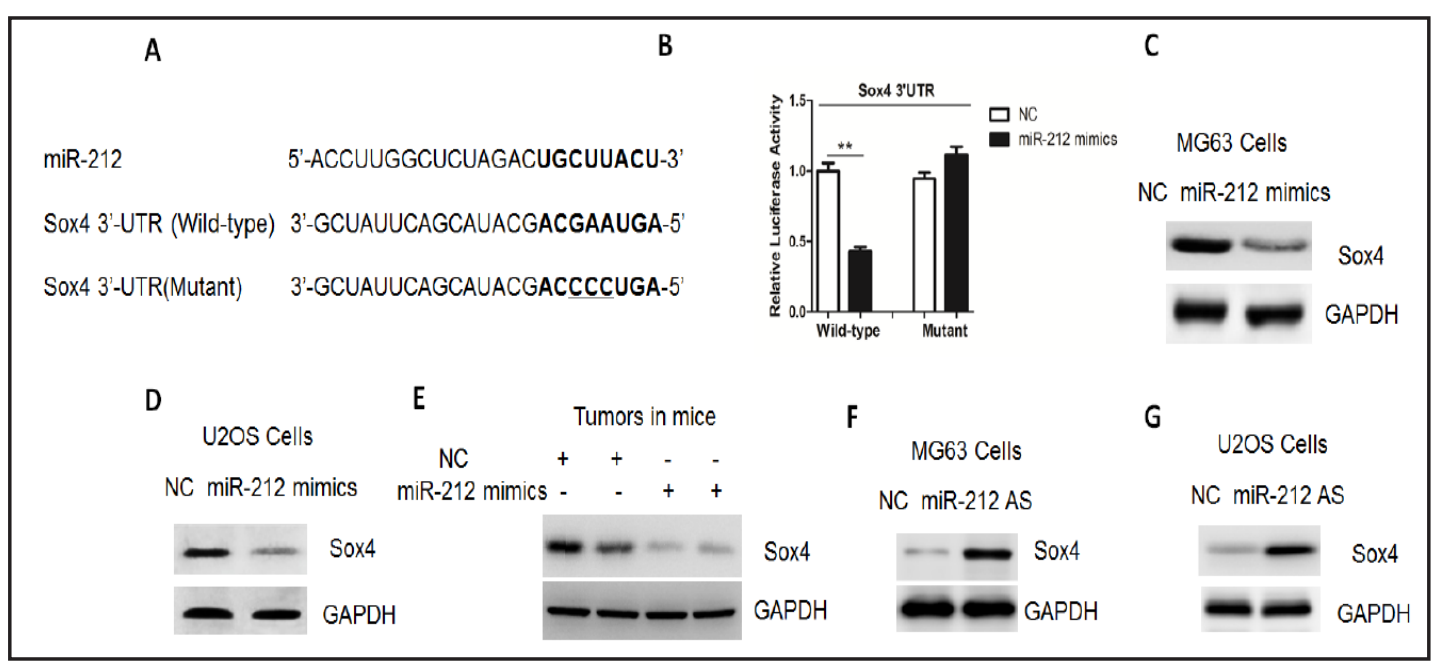

Fig. 5. miR-212 negatively regulates SOX4 expression in osteosarcoma cells. (A) Computer prediction of miR-212 binding sites in the 3'-UTR of human SOX4 gene. Potential binding site was highlighted in bold. (B) Luciferase reporter assays in MG63 cells. Cells were transfected with $200 \mathrm{ng}$ of wild-type 3'-UTR-reporter or mutant constructs together with miR-212 mimics or negative control (NC). (C-D) Western blot analysis of SOX4 expression in MG63 (C) and U2OS (D) cells transfected with miR-212 mimics or negative control (NC). (E) Western blot analysis of SOX4 in tumors from nude mice. (F-G) Western blot analysis of SOX4 expression in MG63 (F) and U2OS (G) cells transfected with miR-212 antisense (AS) or negative control (NC). ${ }^{* *} \mathrm{P}<0.01$ between two groups.

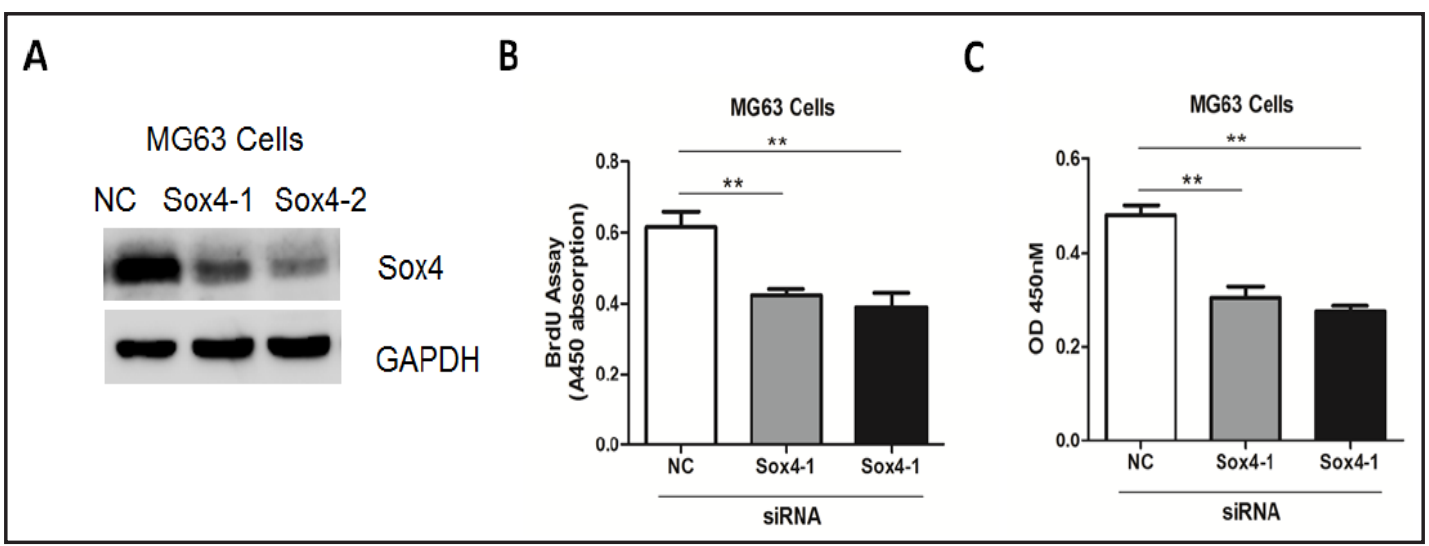

Fig. 6. Sox 4 deficiency showed similar effects with miR-212 overexpression. (A) Representative protein levels of Sox4 were determined by western blot in MG63 cells after transfection of siRNA oligos targeting Sox4 (Sox4-1, Sox4-2) or negative controls (NC). (B-C) The cell proliferation (B) and invasion abilities (C) were determined in MG63 cells with Sox4 depletion.

result, the tumor size, volume and weight were markedly reduced in miR-212-overexpressed tumors compared to control tumors (Fig. 4A-4C), suggesting that miR-212 could also suppress tumor growth in vivo.

miR-212 directly targets the SOX4 in osteosarcoma cells

To clarify the anti-proliferative roles of miR-212, we aimed to identify its regulated targets through bioinformatics software (TargetScan). Among which, we found that the gene encoding sex-determining region Y-box 4 (SOX4) harbored a potential miR-212 binding site (Fig. 5A). Therefore, the 3'-untranslated region (UTR) of SOX4 gene was cloned and inserted into a luciferase reporter construct. Overexpression of miR-212 led to a reduction of luciferase activity carrying the wild-type 3'UTR (Fig. 5B). However, mutation of the potential miR-212 


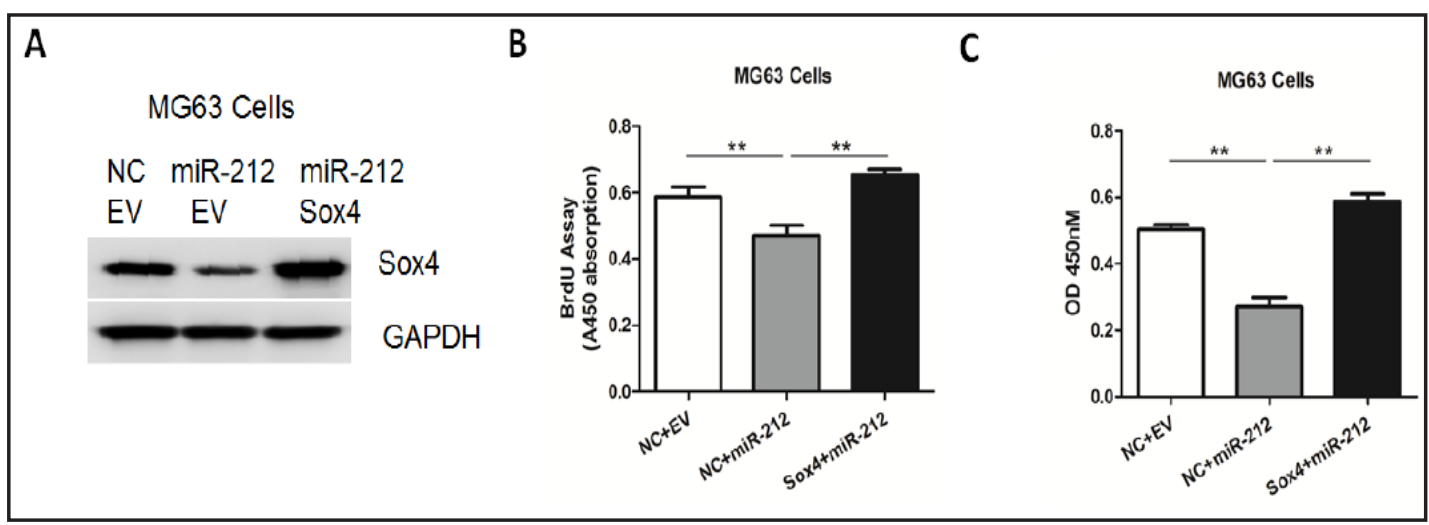

Fig. 7. SOX4 re-introduction reverses the anti-proliferative roles of miR-212. (A) SOX4 protein expression was determined by western blot in MG63 cells. Cells were pre-transfected with miR-212 mimics or negative control (NC) for $24 \mathrm{hr}$, and then transfected with expression plasmids for SOX4 or empty vector (EV) for another $24 \mathrm{hr}$. (B-C) The cell proliferative (B) and invasion abilities (C) were determined in MG63 cells. ** $\mathrm{P}<0.01$ between two groups.

binding site abolished the inhibitory roles of miR-212 (Fig. 5B). Moreover, transfection of miR-212 mimics in osteosarcoma cells resulted in a reduced SOX4 protein expression (Fig. 5C-5D). Besides, protein levels of SOX4 in tumors from nude mice were also reduced by miR212 overexpression (Fig. 5E). In agreement, a dramatic up-regulation of SOX4 was observed in MG63 and U2OS cells with miR-212 inhibition (Fig. 5F-5G). Therefore, our results suggest that SOX4 might be a target of miR-212 in osteosarcoma cells.

\section{Knockdown of Sox 4 showed similar effects with miR-212 overexpression}

To determine the roles of Sox 4 on osteosarcoma cell growth, MG63 cells were transfected with two small interfering RNA (siRNA) oligos targeting Sox4 or negative controls (NC) (Fig. 6A). As expected, knockdown of Sox4 inhibited the proliferation and invasion of MG63 cells (Fig. 6B-6C), suggesting that ablation of Sox4 photocopies the effects of miR-212 overexpression.

SOX4 re-introduction reversed the anti-proliferative roles of miR-212

To further verify the functional connection between miR-212 and SOX4, MG63 cells were transfected with SOX4 expression plasmids after transfection of miR-212 mimics (Fig. 7A). As shown in Figure 7B and 7C, SOX4 re-introduction reversed the anti-proliferative roles of miR-212, underlining the specific importance of the SOX4 for miR-212 action in the cell proliferation and invasion.

\section{Discussion}

In this study, we found that miR-212 expression is down-regulated in osteosarcoma tissues. Forced overexpression of human miR-212 mimics was able to inhibit, while its antisense promoted cell proliferation and invasion in osteosarcoma cells. Besides, the antiproliferative roles of miR-212 were further confirmed in the nude mice. Therefore, our data suggest that miR-212 might be a tumor suppressor in the development and progression of osteosarcoma. However, until now, the mechanisms for the down-regulation of miR-212 remain unknown. Incoronato $\mathrm{M}$ et al. reported that histone modifications could regulate miR212 levels in lung cancer cells [14]. Therefore, we will further investigate whether epigenetic regulation could be involved in the regulation of miR-212 expression in osteosarcoma.

At the molecular level, our results revealed that SOX4 was a direct target of miR-212 in osteosarcoma cells. Recent studies have demonstrated that Sox 4 might be an important 
oncogene contributing to tumor progression [15]. This is suggested by multiple lines of evidence. First, its expression was significantly elevated in multiple human cancers, including tumors of the lung, breast and prostate [16-18]. Second, its overexpression was closely correlated with tumor progression and metastasis $[19,20]$. Third, Sox4 was shown to regulate several key signaling pathways in cancer cells. For instance, Sox4 could regulate $\beta$-catenin/T-cell factor activity and act as an agonist of Wnt signaling in colon cancer [21]. Besides, Sox4 interacted with p53 and inhibits p53-mediated apoptosis in hepatocellular carcinoma [22]. Here, we found that knockdown of Sox4 using siRNA oligos promoted the proliferation and invasion of osteosarcoma cells, suggesting that Sox4 may drive the initiation and (or) progression of osteosarcoma.

Previous studies have revealed that protein levels of Sox 4 were controlled by several miRNAs, such as miR-129-2, miR-138, miR-203 and miR-204 [23-26]. Although the reason why multiple microRNAs regulate a single mRNA remains poorly understood until now, our study together with these reports add a novel mechanism for the up-regulation of Sox4 in human cancers. Therefore, understanding the regulatory mechanism by miR-212/Sox 4 axis might advance our knowledge of osteosarcoma biology, which will help to identify novel gene therapies for its treatment.

\section{Disclosure Statement}

None

\section{Acknowledgment}

This work was supported by The National Natural Science Foundation of China (NO. 81272171; NO. 81372917), Nature Science Foundation Project of CQ CSTC (NO. cstc2013jcyjA10071).

\section{References}

1 Botter SM, Neri D, Fuchs B: Recent advances in osteosarcoma. Curr Opin Pharmacol 2014;16:15-23.

2 Yang J, Zhang W: New molecular insights into osteosarcoma targeted therapy. Curr Opin Oncol 2013;25:398-406.

-3 Sun K, Lai EC: Adult-specific functions of animal microRNAs. Nat Rev Genet 2013;14:535-548.

4 Ameres SL, Zamore PD: Diversifying microRNA sequence and function. Nat Rev Mol Cell Biol 2013;14:475488.

5 Pritchard CC, Cheng HH, Tewari M: MicroRNA profiling: approaches and considerations. Nat Rev Genet 2012;13:358-369.

-6 Ling H, Fabbri M, Calin GA: MicroRNAs and other non-coding RNAs as targets for anticancer drug development. Nat Rev Drug Discov 2013;12:847-865.

7 Kafchinski LA, Jones KB: MicroRNAs in Osteosarcomagenesis. Adv Exp Med Biol 2014;804:119-127.

8 Miao J, Wu S, Peng Z, Tania M, Zhang C: MicroRNAs in osteosarcoma: diagnostic and therapeutic aspects. Tumour Biol 2013;34:2093-2098.

-9 Chen L, Wang Q Wang GD, Wang HS, Huang Y, Liu XM, Cai XH: miR-16 inhibits cell proliferation by targeting IGF1R and the Raf1-MEK1/2-ERK1/2 pathway in osteosarcoma. FEBS Lett 2013;587:1366-1372.

10 Cao ZQ Shen Z, Huang WY: MicroRNA-802 promotes osteosarcoma cell proliferation by targeting p27. Asian Pac J Cancer Prev 2013;14:7081-7084.

11 Jiping Z, Ming F, Lixiang W, Xiuming L, Yuqun S, Han Y, Zhifang L, Yundong S, Shili L, Chunyan C, Jihui J: MicroRNA-212 inhibits proliferation of gastric cancer by directly repressing retinoblastoma binding protein 2. J Cell Biochem 2013;114:2666-2672. 


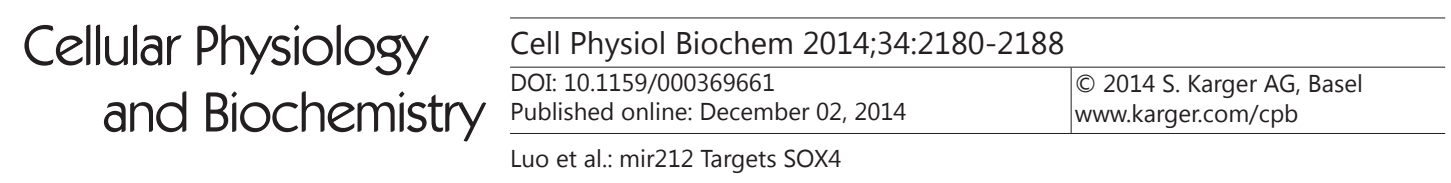

12 Liang X, Zeng J, Wang L, Fang M, Wang Q, Zhao M, Xu X, Liu Z, Li W, Liu S, Yu H, Jia J, Chen C: Histone demethylase retinoblastoma binding protein 2 is overexpressed in hepatocellular carcinoma and negatively regulated by hsa-miR-212. PLoS One 2013;8:e69784.

13 Li Y, Zhang D, Chen C, Ruan Z, Li Y, Huang Y: MicroRNA-212 displays tumor-promoting properties in nonsmall cell lung cancer cells and targets the hedgehog pathway receptor PTCH1. Mol Biol Cell 2012;23:14231434.

-14 Incoronato M, Urso L, Portela A, Laukkanen MO, Soini Y, Quintavalle C, Keller S, Esteller M, Condorelli G: Epigenetic regulation of miR-212 expression in lung cancer. PLoS One 2011;6:e27722.

-15 Jafarnejad SM1, Ardekani GS, Ghaffari M, Li G: Pleiotropic function of SRY-related HMG box transcription factor 4 in regulation of tumorigenesis. Cell Mol Life Sci 2013;70:2677-2696.

16 Castillo SD, Matheu A, Mariani N, Carretero J, Lopez-Rios F, Lovell-Badge R, Sanchez-Cespedes M: Novel transcriptional targets of the SRY-HMG box transcription factor SOX4 link its expression to the development of small cell lung cancer. Cancer Res 2012;72:176-186.

17 Zhang J, Liang Q Lei Y, Yao M, Li L, Gao X, Feng J, Zhang Y, Gao H, Liu DX, Lu J, Huang B: SOX4 induces epithelial-mesenchymal transition and contributes to breast cancer progression. Cancer Res 2012;72:4597-4608.

18 Wang L, Li Y, Yang X, Yuan H, Li X, Qi M, Chang YW, Wang C, Fu W, Yang M, Zhang J, Han B: ERG-SOX4 interaction promotes epithelial- mesenchymal transition in prostate cancer cells. Prostate 2014;74:647 $-658$.

19 Wang L, Zhang J, Yang X, Chang YW, Qi M, Zhou Z, Zhang J, Han B: SOX4 is associated with poor prognosis in prostate cancer and promotes epithelial-mesenchymal transition in vitro. Prostate Cancer Prostatic Dis 2013;16:301-307.

-20 Zhang H, Alberich-Jorda M, Amabile G, Yang H, Staber PB, Di Ruscio A, Welner RS, Ebralidze A, Zhang J, Levantini E, Lefebvre V, Valk PJ, Delwel R, Hoogenkamp M, Nerlov C, Cammenga J, Saez B, Scadden DT, Bonifer C, Ye M, Tenen DG: Sox4 is a key oncogenic target in C/EBP $\alpha$ mutant acute myeloid leukemia. Cancer Cell 2013;24:575-588.

21 Lee AK, Ahn SG, Yoon JH, Kim SA: Sox4 stimulates ß-catenin activity through induction of CK2. Oncol Rep 2011;25:559-565.

22 Hur W, Rhim H, Jung CK, Kim JD, Bae SH, Jang JW, Yang JM, Oh ST, Kim DG, Wang HJ, Lee SB, Yoon SK: SOX4 overexpression regulates the p53-mediated apoptosis in hepatocellular carcinoma: clinical implication and functional analysis in vitro. Carcinogenesis 2010;31:1298-1307.

23 Kang M, Li Y, Liu W, Wang R, Tang A, Hao H, Liu Z, Ou H: miR-129-2 suppresses proliferation and migration of esophageal carcinoma cells through downregulation of SOX4 expression. Int J Mol Med 2013;32:51-58.

24 Yeh YM, Chuang CM, Chao KC, Wang LH: MicroRNA-138 suppresses ovarian cancer cell invasion and metastasis by targeting SOX4 and HIF- $1 \alpha$. Int J Cancer 2013;133:867-878.

-25 Huang YW, Kuo CT, Chen JH, Goodfellow PJ, Huang TH, Rader JS, Uyar DS: Hypermethylation of miR-203 in endometrial carcinomas. Gynecol Oncol 2014;133:340-345.

-26 Zhou X, Li L, Su J, Zhang G: Decreased miR-204 in H. pylori-associated gastric cancer promotes cancer cell proliferation and invasion by targeting SOX4. PLoS One 2014;9:e101457. 